\title{
Inhibition of $\mathrm{p38}$ mitogen-activated protein kinase potentiates the apoptotic effect of berberine/tumor necrosis factor-related apoptosis-inducing ligand combination therapy
}

\author{
ALAA REFAAT $^{1-3}$, SHERIF ABDELHAMED ${ }^{1}$, IKUO SAIKI ${ }^{1}$ and HIROAKI SAKURAI ${ }^{2}$ \\ ${ }^{1}$ Division of Pathogenic Biochemistry, Department of Bioscience, Institute of Natural Medicine; \\ ${ }^{2}$ Division of Cancer Cell Biology, Graduate School of Pharmaceutical Sciences, University of Toyama, Toyama 930-0194, \\ Japan; ${ }^{3}$ Center for Aging and Associated Diseases, Zewail City of Science and Technology, Giza 12588, Egypt
}

Received November 6, 2014; Accepted May 29, 2015

DOI: $10.3892 / \mathrm{ol} .2015 .3494$

\begin{abstract}
It was previously reported that berberine (BBR) and tumor necrosis factor (TNF)-related apoptosis-inducing ligand (TRAIL) exhibited a synergistic apoptotic effect on triple negative breast cancer (TNBC) cells. In addition, the BBR/TRAIL combination treatment sensitized TRAIL-resistant TNBC cells to TRAIL. The aim of the present study was to investigate a novel pathway for enhancing the apoptotic effect of BBR/TRAIL through mitogen-activated protein kinases (MAPKs). Selective inhibitors and small interfering RNAs were utilized to understand the role of p38 MAPK in this pathway. The results demonstrated that p38 MAPK was activated in response to the combination therapy in TRAIL-resistant TNBC cells. In addition, it was revealed that the inhibition of p38 enhanced apoptosis in epidermal growth factor receptor (EGFR)-overexpressing MDA-MB-468 TNBC cells and EGFR-mutant PC-9 non-small-cell lung carcinoma cells, which was associated with the downregulation of EGFR serine phosphorylation. Viability assays for these two cell lines also confirmed the significant reduction of cell viability following p38 inhibition in BBR/TRAIL-treated cells. In conclusion, the present study provided novel evidence for the role of p38 in suppressing BBR/TRAIL-mediated apoptosis and its association with EGFR, which may explain the mechanism of treatment resistance in certain types of cancer.
\end{abstract}

Correspondence to: Dr Alaa Refaat, Center for Aging and Associated Diseases, Zewail City of Science and Technology, 26 July Axis, Sheikh Zayed District, 6th of October City, Giza 12588, Egypt E-mail: arefaat@zewailcity.edu.eg

Professor Hiroaki Sakurai, Division of Cancer Cell Biology, Graduate School of Pharmaceutical Sciences, University of Toyama, 2630 Sugitani, Toyama-shi, Toyama 930-0194, Japan

E-mail: hsakurai@pha.u-toyama.ac.jp

Key words: apoptosis, berberine, epidermal growth factor receptor, mitogen-activated protein kinase, tumor necrosis factor-related apoptosis-inducing ligand

\section{Introduction}

Berberine (BBR) is an isoquinoline alkaloid found in the rhizome of numerous valuable medicinal plants (1). BBR has been reported to exhibit anti-inflammatory activity via the inhibition of transforming growth factor- $\beta$-activated kinase 1 (TAK1) (2). TAK1 is an important mediator of nuclear factor- $\kappa \mathrm{B}$ activation, which in turn has important roles in cell survival and inflammation (3).

Tumor necrosis factor (TNF)-related apoptosis-inducing ligand (TRAIL) has potential antitumor activity, due to its high selectivity for various types of cancer cells over normal cells (4). However, the clinical efficacy of TRAIL has been limited due to primary or acquired resistance $(5,6)$. TRAIL-based combination therapy approaches have therefore been introduced as a novel strategy against resistance $(1,7,8)$. Of note, several studies have reported the contribution of the activated epidermal growth factor (EGF) receptor (EGFR) pathway in TRAIL-resistance $(9,10)$. TRAIL activates TAK1 in tumor cells (11), which may result in the phosphorylation of downstream mitogen-activated protein kinases (MAPKs), most importantly p38 and extracellular signal-regulated kinase (ERK), and the subsequent regulation of the EGFR pathway $(12,13)$.

EGFR (ErbB-1) is a member of the ErbB family of receptors, which is activated through the binding of its specific ligands (14). In canonical EGFR activation, EGF binds to EGFR to induce the preferential phosphorylation of tyrosine residues, which subsequently results in the internalization of EGFR into the cytoplasm to enhance the survival and proliferation of cells (14). By contrast, the TNF- $\alpha$ ligand, a member of the TNF family, binds to its relevant receptor to induce the TAK1-mediated activation of MAPKs and the subsequent phosphorylation of serine/threonine residues of EGFR. This non-canonical pathway of MAPKs/EGFR may eventually result in the p38/serine-dependent internalization of EGFR (14). However, the functional role of the p38/serine-dependent internalization remains to be fully elucidated $(12,15)$.

A previous study demonstrated the efficiency of a BBR/TRAIL combination therapy against triple negative breast cancer (TNBC) cells as well as its effect on the sensitization of TRAIL-resistant TNBC cells to TRAIL (11). The 
aim of the present study was to investigate a novel pathway for potentiating the apoptotic effect of BBR/TRAIL combination therapy through p38 MAPKs.

\section{Materials and methods}

Antibodies and reagents. Phospho-specific antibodies against p38 (Thr-180, Tyr-182; rabbit anti-human; cat. no., 9211), ERK (Thr-202, Tyr-204; rabbit anti-human; cat. no. 9101), and EGFR (Tyr-1068, Thr-669 and Ser-1046/1047; mouse anti-human; cat. nos. 2236, 3056 and 2238, respectively) as well as antibodies against poly-(adenosine diphosphate-ribose) polymerase 1 (PARP-1; rabbit anti-human; cat. no. 9542) and caspase-3 (rabbit anti-human; cat. no. 9662) were purchased from Cell Signaling Technology, Inc. (Danvers, MA, USA). Antibodies against EGFR (1005; rabbit anti-human; cat. no. sc-03) and $\beta$-actin (C-11; goat anti-human; cat. no. sc-1615) were obtained from Santa Cruz Biotechnology, Inc. (Dallas, TX, USA). All antibodies were polyclonal, with the exception of EGFR (Tyr-1068) that was monoclonal. Primary antibodies were used at 1:1,000 dilution. Recombinant human TRAIL Apo II ligand was obtained from PeproTech, Inc. (Rocky Hill, NJ, USA). SB203580, U0126 and PD153035, which are inhibitors of p38, ERK and EGFR tyrosine kinase, respectively, were obtained from Merck Biosciences (Danvers, MA, USA), while 5Z-7-oxozeaenol, a selective TAK1 inhibitor, was obtained from Chugai Pharmaceutical Co., Ltd. (Tokyo, Japan). BBR chloride was purchased from Wako Pure Chemical Industries, Ltd. (Osaka, Japan). All chemical inhibitors, in addition to, BBR chloride were dissolved in dimethyl sulfoxide (Wako Pure Chemical Industries, Ltd.).

Cell cultures. MDA-MB-468 cells (ATCC, Rockville, MD, USA) were maintained in Dulbecco's modified Eagle's medium (DMEM; Invitrogen Life Technologies, Carlsbad, CA, USA) supplemented with 10\% fetal calf serum (Invitrogen Life Technologies), $2 \mathrm{mM}$ glutamine (Life Technologies, Gaithersburg, MD, USA), $100 \mathrm{U} / \mathrm{ml}$ penicillin and $100 \mu \mathrm{g} / \mathrm{ml}$ streptomycin (Life Technologies) at $37^{\circ} \mathrm{C}$ in a humidified atmosphere of $5 \% \mathrm{CO}_{2}$. PC-9 cells were provided by Dr Kiura (Okayama University, Okayama, Japan) and were maintained in RPMI-1640 medium (Invitrogen Life Technologies), with identical supplements and under identical conditions.

Cell viability assay. A WST-1 Cell Counting kit (Dojindo, Kumamoto, Japan) was used to detect cell viability. In brief, cell suspensions in DMEM supplemented with $10 \%$ fetal calf serum were seeded into a 96 -well plate $\left(5-8 \times 10^{3} / 70 \mu 1 /\right.$ well $)$ and incubated at $37^{\circ} \mathrm{C}$ in a humidified atmosphere of $5 \% \mathrm{CO}_{2}$. Following $24 \mathrm{~h}$ of incubation, $10 \mu \mathrm{l} \mathrm{FBS}$-supplemented DMEM containing the inhibitors at specified concentrations were added to the wells. After $30 \mathrm{~min}, 10 \mu \mathrm{l}$ medium containing BBR (Wako Pure Chemical Industries, Ltd.) was added to the respective wells and incubated at $37^{\circ} \mathrm{C}$ for $90 \mathrm{~min}$, followed by $10 \mu 1$ medium containing TRAIL (PeproTech, Inc., Rocky Hill, NJ, USA). Plates were then incubated for $24 \mathrm{~h}$ at $37^{\circ} \mathrm{C}$. WST-1 solution $(10 \mu \mathrm{l})$ was added to each well $2 \mathrm{~h}$ prior to the end of the experiment. Absorbance was measured at $450 \mathrm{~nm}$ using a microplate reader (Multiskan Plus Model 355 Microplate Reader; Thermo Labsystems, Helsinki, Finland). Cell viability

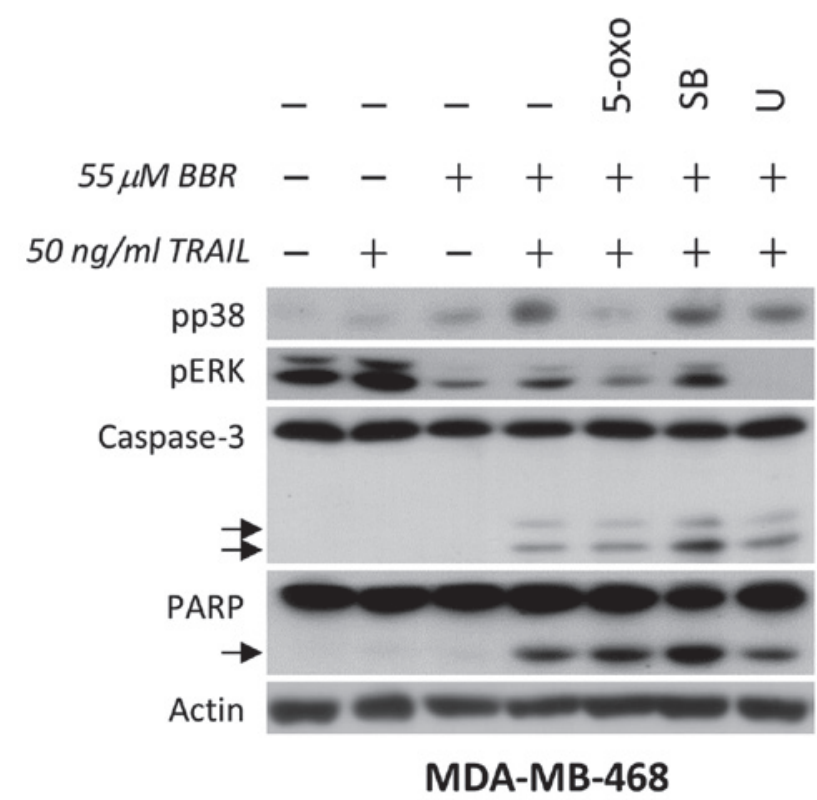

Figure 1. p38 counteracts the apoptotic effect mediated by BBR/TRAIL combination therapy in human breast cancer cells. TRAIL-resistant MDA-MB-468 cells were pretreated with dimethyl sulfoxide, $0.3 \mu \mathrm{M}$ 5-oxo (transforming growth factor- $\beta$-activated kinase 1 ), $10 \mu \mathrm{M} \mathrm{SB}$ (p38 inhibitor) or $5 \mu \mathrm{M} \mathrm{U}$ (ERK inhibitor) for $30 \mathrm{~min}$, followed by incubation with $55 \mu \mathrm{M}$ BBR for $90 \mathrm{~min}$. Cells were then further incubated in the presence or absence of recombinant human TRAIL $(50 \mathrm{ng} / \mathrm{ml})$ for $3 \mathrm{~h}$. Whole cell lysates were analyzed by immunoblotting for the indicated proteins. TRAIL, tumor necrosis factor-related apoptosis-inducing ligand; BBR, berberine; 5-oxo, 5Z-7-oxozeaenol; SB, SB203580; U, U0126; ERK, extracellular signal-regulated kinase; PARP, poly-(adenosine diphosphate-ribose) polymerase 1.

was determined from the absorbance of soluble formazan dye generated by living cells.

Transfection of small interfering RNAs (siRNAs). Duplex siRNAs were synthesized at Hokkaido System Science Co.,Ltd. (Sapporo, Japan). The target sequences were as follows: $\mathrm{p} 38 \alpha$, 5'-GCAUUACAACCAGACAGUUGAUAUU-3'; and firefly luciferase (GL2), 5'-CGUACGCGGAAUACUUCGA-3'. MDA-MB-468 cells were transfected with siRNAs in a final concentration of $50 \mathrm{nM}$ using lipofectamine (Life Technologies). At $72 \mathrm{~h}$ post-transfection, the cells were treated.

Immunoblotting. Following stimulation, whole-cell lysates were prepared as described previously (16). Cell lysates were resolved by $7.5,10$ or $12.5 \%$ sodium dodecyl sulfate-polyacrylamide gel electrophoresis and transferred to an Immobilon-P nylon membranes (EMD Millipore, Billerica, MA, USA). The membranes were treated with BlockAce (Dainippon Pharmaceutical Co., Ltd., Suita, Japan) and probed with primary antibodies (dilution, 1:1,000). The antibodies were then detected using polyclonal goat anti-rabbit (cat. no. P0448), polyclonal rabbit anti-mouse (cat. no. P0260) or polyclonal rabbit anti-goat (cat. no. P0449) horseradish peroxidase-conjugated immunoglobulin G antibody (dilution, 1:2,000; Dako, Carpinteria, CA, USA) and visualized using an enhanced chemiluminescence system (Amersham Biosciences, Piscataway, NJ, USA). Certain antibody reactions were performed using in Can Get Signal ${ }^{\circledR}$ immunostain solution (Toyobo Co., Ltd, Osaka, Japan). 
A

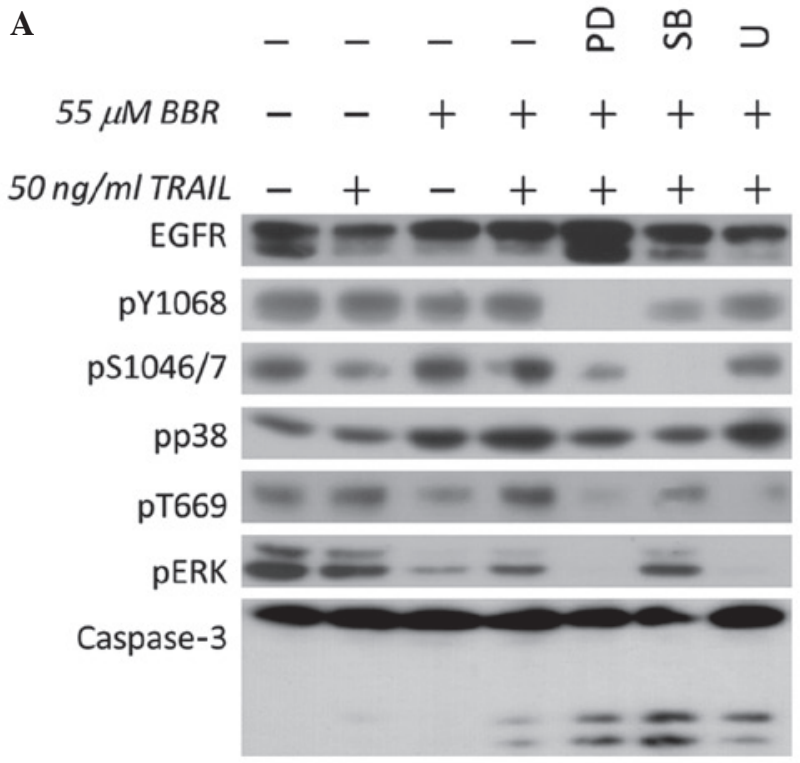

PARP

Actin
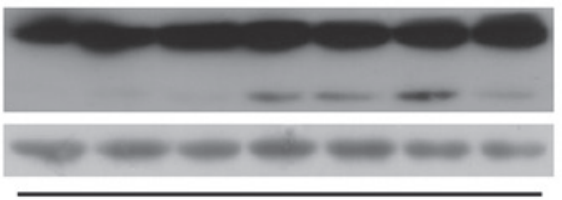

MDA-MB-468
B

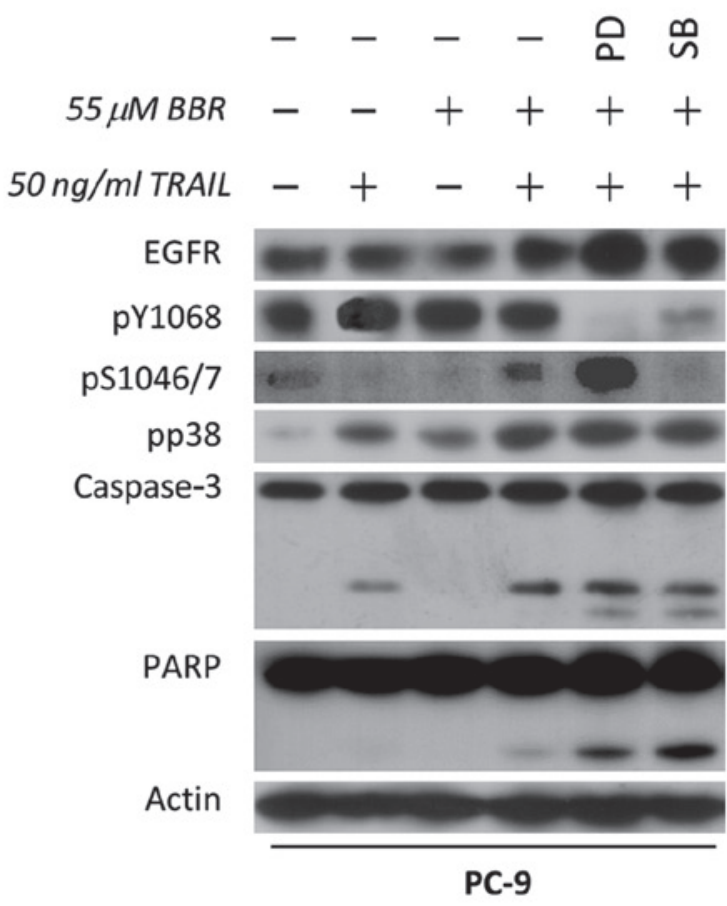

Figure 2. Crosstalk between EGFR and the apoptotic effect mediated by BBR/TRAIL combination therapy in EGFR-expressing breast cancer cells. (A) EGFR-overexpressing MDA-MB-468 and (B) mutant EGFR-overexpressing PC-9 cells were pretreated with dimethyl sulfoxide, $1 \mu$ M PD (EGFR tyrosine kinase inhibitor), $10 \mu \mathrm{M} \mathrm{SB}$ (p38 inhibitor) or $5 \mu \mathrm{M} \mathrm{U}$ (ERK inhibitor) for $30 \mathrm{~min}$, followed by incubation with $55 \mu \mathrm{M}$ BBR for 90 min. Cells were then further incubated in the presence or absence of $50 \mathrm{ng} / \mathrm{ml}$ recombinant human TRAIL for $3 \mathrm{~h}$. Whole cell lysates were analyzed by immunoblotting for the indicated proteins. TRAIL, tumor necrosis factor-related apoptosis-inducing ligand; BBR, berberine; EGFR, epidermal growth factor receptor; PD, PD153035; SB, SB203580; U, U0126; ERK, extracellular signal-regulated kinase; PARP, poly-(adenosine diphosphate-ribose) polymerase 1.

Statistical analysis. Data are presented as the mean \pm standard deviation of 3 experiments. The statistical analysis was performed using JMP software (version 10; SAS Institute Inc., Cary, NC, USA). The statistical significance was determined by applying the Bonferroni method (compared to BBR/TRAIL). $\mathrm{P}<0.05$ was considered to indicate a statistically significant difference.

\section{Results}

TRAILenhances BBR-mediatedp38activation and counteracts $B B R$-mediated pERK downregulation in a TAK1-dependent manner. In light of the previous findings of BBR and TRAIL studies $(1,17)$, the present study aimed to investigate the effect of the BBR/TRAIL combination therapy on MAPK activation in EGFR-overexpressing breast cancer cells. The results demonstrated that TRAIL alone markedly upregulated ERK phosphorylation and slightly upregulated $\mathrm{p} 38$ phosphorylation in MDA-MB-468 cells (Fig. 1). This effect was not associated with any changes in apoptosis, which may be due to the resistance of MDA-MB-468 cells to TRAIL (Fig. 1, lane 2). By contrast, BBR decreased ERK phosphorylation and markedly increased p38 phosphorylation (Fig. 1). In addition, the BBR/TRAIL combination further enhanced p38 phosphorylation and decreased ERK phosphorylation. TAK1 inhibition using the specific inhibitor 5Z-7-oxozeaenol demonstrated an inhibition of the combination therapy-mediated upregulation of p38; comparably, decreased ERK phosphorylation was observed following 5Z-7-oxozeaenol treatment. This indicated that TAK1 was involved in the regulation of the phosphorylation of p38 and ERK in MDA-MB-468 cells. However, TAK1 inhibition did not induce any changes in combination therapy-mediated apoptosis (Fig. 1, lane 5 vs. 4). In order to further demonstrate the effect of $\mathrm{p} 38$ and ERK inhibition on cell apoptosis, cells were pretreated with specific inhibitors, SB203580 and U0126, respectively. The results revealed that p38 inhibition enhanced apoptosis in cells treated with BBR/TRAIL combination therapy, whereas ERK inhibition did not exhibit notable effects on apoptosis (Fig. 1).

p38 MAPK rescues cancer cells from BBR/TRAIL combination therapy. In order to confirm the efficient inhibition of p38 in EGFR-expressing cells, the previous experiment was repeated with EGFR tyrosine kinase inhibition instead of TAK1 inhibition. EGFR tyrosine kinase is well-known to be crucial for cell survival and proliferation (18). Although blocking the EGFR tyrosine kinase may indirectly block ERK and EGFR threonine phosphorylation, the present results demonstrated that p38 inhibition downregulated EGFR serine phosphorylation as well as enhanced apoptosis compared with that of EGFR tyrosine kinase inhibition. In addition, ERK inhibition did not promote apoptosis in MDA-MB-468 cells (Fig. 2A). This confirmed that $\mathrm{p} 38$, but not ERK, may contribute to cell apoptosis in EGFR-expressing MDA-MB-468 cells. In order to confirm these results, the effects of the EGFR tyrosine kinase inhibitor and p38 inhibitor were examined in EGFR-mutant 

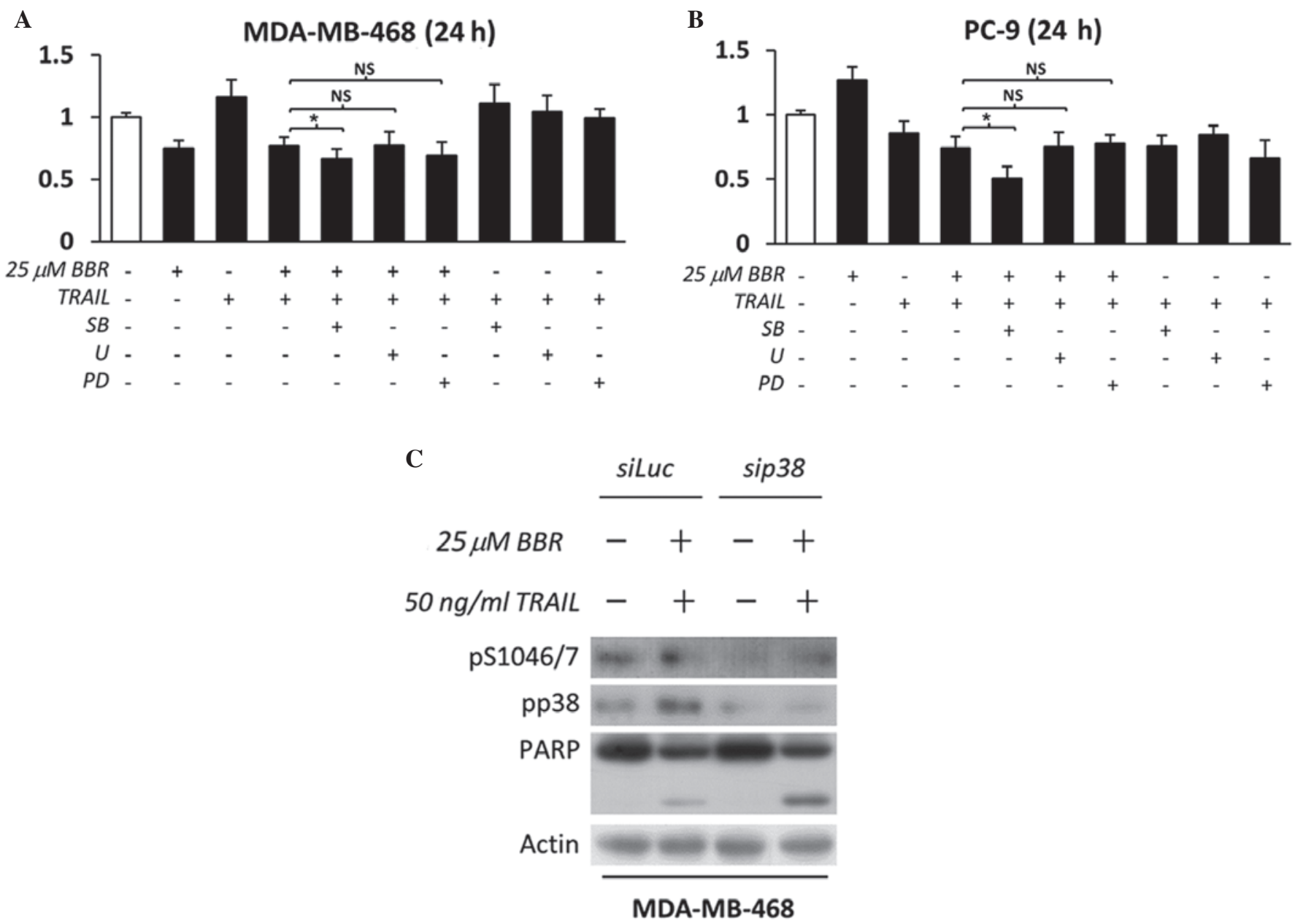

Figure 3.p38 inhibition enhances the apoptotic effect mediated by BBR/TRAIL combination therapy in EGFR-expressing cancer cells. (A) EGFR-overexpressing MDA-MB-468 and (B) mutant EGFR-overexpressing PC-9 cells were pretreated with dimethyl sulfoxide, $1 \mu$ M PD (EGFR tyrosine kinase inhibitor), $10 \mu \mathrm{M} \mathrm{SB}$ (p38 inhibitor) or $5 \mu \mathrm{M}$ U (extracellular signal-regulated kinase inhibitor) for 30 min, followed by incubation with $25 \mu \mathrm{M}$ BBR for 90 min. Cells were then further incubated in the presence or absence of $50 \mathrm{ng} / \mathrm{ml}$ recombinant human TRAIL for $24 \mathrm{~h}$ in the presence or absence of specific inhibitors. Absorbance was measured following incubation with WST-1 reagent and cell viability was calculated as a percentage relative to the untreated control cells. Data are expressed as the mean \pm standard deviation of triplicate experiments. ${ }^{*} \mathrm{P}<0.05$ between groups; NS, not significant. (C) MDA-MB-468 cells were transfected with Luc (control) or p38 $\alpha$ siRNA in the presence or absence of BBR/TRAIL. Whole cell lysates were analyzed by immunoblotting for the indicated proteins. TRAIL, tumor necrosis factor-related apoptosis-inducing ligand; BBR, berberine; EGFR, epidermal growth factor receptor; siRNA, small interfering RNAs; PD, PD153035; SB, SB203580; U, U0126; PARP, poly-(adenosine diphosphate-ribose) polymerase 1; Luc, luciferase.

non-small-cell lung carcinoma (NSCLC) PC-9 cells, the results of which demonstrated a comparable pattern to that of the EGFR-expressing breast cancer cells (Fig. 2B). The consequences of enhancing cellular apoptosis following combination therapy were demonstrated using cell viability assays in these EGFR-overexpressing cell lines; MDA-MB-468 and PC-9. In this experiment, cells were exposed to a lower concentration of BBR, in addition to TRAIL, and the specific inhibitors for extended time periods in order to monitor their significance on cell viability. As shown in Fig. 3A and B, only p38 inhibition in addition to BBR/TRAIL therapy induced a significant decrease in cell viability, compared to ERK inhibition or EGFR inhibition. In addition, the specificity of p38 action was confirmed using specific knockdown of p38 with siRNA. The results demonstrated that $\mathrm{p} 38$ knockdown induced EGFR serine downregulation, which resulted in the cleavage of caspase-substrate, PARP-1 (Fig. 3C); this therefore confirmed the value of using p38 inhibition in conjunction with BBR/TRAIL combination therapy in EGFR-expressing cancer cells.

\section{Discussion}

A previous study demonstrated the role of BBR in synergizing TRAIL action in TRAIL-sensitive MDA-MB-231 TNBC cells, as well as sensitizing the TRAIL-resistant MDA-MB-468 TNBC cells to TRAIL (1). In addition to the difference in TRAIL sensitivity pattern, MDA-MB-468, and not MDA-MB-231 cells, were reported to preferentially overexpress EGFR (1). Furthermore, through comparing data from the publicly available microarray (GSE41313; http://www. ncbi.nlm.nih.gov/geo/query/acc.cgi?acc=GSE41313), it was revealed that MDA-MB-468 exhibited the highest expression of EGFR among other breast cancer cells (data not shown). In the present study, the role of MAPK in cellular apoptosis was investigated in EGFR-expressing cells. MDA-MB-468 cells were primarily used in the present study, with a secondary focus on PC-9 EGFR-mutant NSCLC cells. The selected cell lines facilitated the exploration of the present findings regardless of the EGFR status. 
In concurrence with previous studies $(12,14,19)$, the present study suggested that the role of p38 in EGFR serine phosphorylation is of increasing importance for cell survival. In order to explore the role of p38 in BBR/TRAIL-mediated apoptosis, independent of the EGFR-expression status, the present study used MDA-MB-468 and PC-9 cells; the results of which confirmed the role of p38 in counteracting BBR/TRAIL-mediated apoptosis. The role of p38 in EGFR serine was not thoroughly investigated in the present study; however, these results provided certain evidence for this reproducible association. It was therefore suggested that $\mathrm{p} 38$ may counteract the BBR/TRAIL-mediated apoptosis as well as suppress the apoptotic effect of other therapies.

The role of $\mathrm{p} 38$ in cell death/survival has been a controversial issue for decades. We believe that the type, strength and duration of specific stimuli towards p38 may significantly affect the eventual outcome experienced by the cell. However, in cancer cells expressing EGFR, growing evidence indicates the prominence of the p38-EGFR serine axis on cell survival. Our recent studies demonstrated the role of various stimuli on the activation of this survival axis $(12,18)$. Notably, in a recent study, we demonstrated a similar apoptotic response subsequent to inhibiting the p38-EGFR serine axis (14). Therefore, studies involving the use of specific p38 inhibitors may be of potential future use in anticancer combination therapies.

\section{Acknowledgements}

The present study was supported, in part, by a short-term fellowship grant from the Science and Technology Development Fund, Egypt (no. 6093).

\section{References}

1. Refaat A, Abdelhamed S, Yagita H, Inoue H, Yokoyama S Hayakawa Y and Saiki I: Berberine enhances tumor necrosis factor-related apoptosis-inducing ligand-mediated apoptosis in breast cancer. Oncol Lett 6: 840-844, 2013.

2. Zhang Y, Li X, Zhang Q, Li J, Ju J, Du N, Liu X, Chen X, Cheng F, Yang L, et al: Berberine hydrochloride prevents postsurgery intestinal adhesion and inflammation in rats. J Pharmacol Exp Ther 349: 417-426, 2014.

3. Sakurai H: Targeting of TAK1 in inflammatory disorders and cancer. Trends Pharmacol Sci 33: 522-530, 2012.

4. Keane MM,Ettenberg SA, Nau MM, Russell EK and Lipkowitz S: Chemotherapy augments TRAIL-induced apoptosis in breast cell lines. Cancer Res 59: 734-741, 1999.

5. Tolcher AW, Mita M, Meropol NJ, von Mehren M, Patnaik A, Padavic K, Hill M, Mays T, McCoy T, Fox NL, et al: Phase I pharmacokinetic and biologic correlative study of mapatumumab, a fully human monoclonal antibody with agonist activity to tumor necrosis factor-related apoptosis-inducing ligand receptor-1. J Clin Oncol 25: 1390-1395, 2007.
6. Dyer MJ, MacFarlane M and Cohen GM: Barriers to effective TRAIL-targeted therapy of malignancy. J Clin Oncol 25: 4505-4506, 2007.

7. Abdelhamed S, Yokoyama S, Refaat A, Ogura K, Yagita H, Awale S and Saiki I: Piperine enhances the efficacy of TRAIL-based therapy for triple-negative breast cancer cells. Anticancer Res 34: 1893-1899, 2014.

8. Refaat A, Abd-Rabou A and Reda A: TRAIL combinations: The new 'trail' for cancer therapy (Review). Oncol Lett 7: 1327-1332, 2014.

9. Xu L, Zhang Y, Liu J, Qu J, Hu X, Zhang F, Zheng H, Qu X and Liu Y: TRAIL-activated EGFR by Cbl-b-regulated EGFR redistribution in lipid rafts antagonises TRAIL-induced apoptosis in gastric cancer cells. Eur J Cancer 48: 3288-3299, 2012.

10. Xu L, Qu X, Li H, Li C, Liu J, Zheng H and Liu Y: Src/caveolin-1-regulated EGFR activation antagonizes TRAIL-induced apoptosis in gastric cancer cells. Oncol Rep 32: 318-324, 2014.

11. Choo MK, Kawasaki N, Singhirunnusorn P, Koizumi K, Sato S, Akira S, Saiki I and Sakurai H: Blockade of transforming growth factor-beta-activated kinase 1 activity enhances TRAIL-induced apoptosis through activation of a caspase cascade. Mol Cancer Ther 5: 2970-2976, 2006.

12. Nishimura M, Shin MS, Singhirunnusorn P, Suzuki S, Kawanishi M, Koizumi K, Saiki I and Sakurai H: TAK1-mediated serine/threonine phosphorylation of epidermal growth factor receptor via p38/extracellular signal-regulated kinase: NF- $\{$ kappa\}B-independent survival pathways in tumor necrosis factor alpha signaling. Mol Cell Biol 29: 5529-5539, 2009.

13. Wang C, Chen T, Zhang N, Yang M, Li B, Lü X, Cao X and Ling C: Melittin, a major component of bee venom, sensitizes human hepatocellular carcinoma cells to tumor necrosis factor-related apoptosis-inducing ligand (TRAIL)-induced apoptosis by activating CaMKII-TAK1-JNK/p38 and inhibiting IkappaBalpha kinase-NFkappaB. J Biol Chem 284: 3804-3813, 2009.

14. Refaat A, Aminullah, Zhou Y, Kawanishi M, Tomaru R, Abdelhamed S, Shin MS, Koizumi K, Yokoyama S, Saiki I and Sakurai H: Role of tyrosine kinase-independent phosphorylation of EGFR with activating mutation in cisplatin-treated lung cancer cells. Biochem Biophys Res Commun 458: 856-861, 2015.

15. Tong J, Taylor P, Peterman SM, Prakash A and Moran MF: Epidermal growth factor receptor phosphorylation sites Ser991 and Tyr998 are implicated in the regulation of receptor endocytosis and phosphorylations at Ser1039 and Thr1041. Mol Cell Proteomics 8: 2131-2144, 2009.

16. Refaat A, Zhou Y, Suzuki S, Takasaki I, Koizumi K, Yamaoka S, Tabuchi Y, Saiki I and Sakurai H: Distinct roles of transforming growth factor-beta-activated kinase 1 (TAK1)-c-Rel and interferon regulatory factor 4 (IRF4) pathways in human T cell lymphotropic virus 1-transformed T helper 17 cells producing interleukin-9. J Biol Chem 286: 21092-21099, 2011.

17. Lee SJ, Noh HJ, Sung EG, Song IH, Kim JY, Kwon TK and Lee TJ: Berberine sensitizes TRAIL-induced apoptosis through proteasome-mediated downregulation of c-FLIP and Mcl-1 proteins. Int J Oncol 38: 485-492, 2011.

18. Zaidi SF, Refaat A, Zhou Y, Sualeh Muhammad J, Shin MS, Saiki I, Sakurai H and Sugiyama T: Helicobacter pylori induces serine phosphorylation of EGFR via novel TAK1-p38 activation pathway in an HB-EGF-independent manner. Helicobacter: Feb 23, 2015 (Epub ahead of print).

19. Zhou Y, Tanaka T, Sugiyama N, Yokoyama S, Kawasaki Y, Sakuma T, Ishihama Y, Saiki I and Sakurai H: p38-Mediated phosphorylation of Eps15 endocytic adaptor protein. FEBS Lett 588: 131-137, 2014. 